\section{Klassifikatorische Diagnostik von Störungen durch psychotrope Substanzen}

Zusammenfassung: Die klassifikatorische Diagnostik von Störungen durch psychotrope Substanzen hat durch die Veröffentlichung von ICD-10 und DSM-III, DSM-III-R und DSM-IV eine substanzielle Verbesserung erfahren. In dem Beitrag werden einige der konzeptuellen Veränderungen kritisch diskutiert und die Klassifikationskonzepte von ICD-10 und DSM-IV gegenübergestellt. Auf multiaxiale diagnostische Ansätze wird gesondert eingegangen.

Schlüsselwörter: ICD-10 - DSM-IV - OPD - Komorbidität Multiaxiale Diagnostik

The Classification of Disorders due to Psychoactive Substance Use: The classification of disorders due to psychoactive substance use has been improved by the publication of ICD-10, DSM-III, DSM-III-R and DSM-IV. The paper provides an overview of the conceptual innovations including a crosswalk ICD-10 vs. DSM-IV and a discussion of multiaxial approaches.

Key words: ICD-10 - DSM-IV - OPD - Comorbidity - Multiaxial Diagnosis

\section{Einleitung}

Klassifikatorische Suchtdiagnostik gehörte bis zur Veröffentlichung der neueren operationalisierten Klassifikationssysteme eher $z u$ den vernachlässigten klinischen bzw. Forschungsbereichen [1,2,3]. Durch die Entwicklung von ICD-10 und DSM-III, DSM-III-R und DSM-IV wurden auch für den Suchtbereich wesentliche Forschungsentwicklungen angestoßen und dabei vor allem die Reliabilität und Validität der vorliegenden Klassifikationsansätze überprüft (vgl. zur ICD-10 auch [3-5]). Wesentlich stimuliert wurden die Forschungsbemühungen darüber hinaus durch breit angelegte epidemiologische Studien der 80er Jahre (z.B. [6]), durch versorgungsepidemiologische Untersuchungsansätze (z.B. [7]), die Komorbiditätsforschung (z.B. $[8,9])$ sowie schließlich die Entwicklung suchtspezifischer diagnostischer Instrumente, wie etwa dem Addiction Severity Index [10].

Suchttherapie $2001 ; 2: 2-8$

(c) Georg Thieme Verlag Stuttgart · New York ISSN 1439-9903
Harald J. Freyberger ${ }^{1}$, Rolf-Dieter Stieglitz ${ }^{2}$

${ }^{1}$ Klinik und Poliklinik für Psychiatrie und Psychotherapie der Ernst-Moritz-Arndt Universität Greifswald im Klinikum Stralsund

2 Psychiatrische Universitätspoliklinik Basel

Im Folgenden sollen einige grundlegende Ansätze der operationalisierten Diagnostik der Störungen durch psychotrope Substanzen vorgestellt und diskutiert werden.

\section{Missbrauch, Abhängigkeit und andere Störungskonzepte in ICD-10 und DSM-IV}

Das Konzept der ICD-10 [11,12] und des DSM-IV [13], den Missbrauch oder schädlichen Gebrauch von Abhängigkeit im Sinne distinkter diagnostischer Kategorien zu unterscheiden, geht auf das dichotome Konzept von Edwards und Gross [14] zurück. Hinsichtlich der definierten diagnostischen Merkmale sind beide Systeme fast identisch, sie unterscheiden sich vor allem durch die explizite Berücksichtigung sozialer Faktoren bzw. des sozialen Funktionsniveaus im DSM-IV bei ihren Missbrauchs- und Abhängigkeitsbegriffen (vgl. Tab. 1 und 2). In der ICD-10 wurden soziale Faktoren wegen ihrer unter-

Tab. 1 Missbrauchsdefinitionen in ICD-10 und DSM-IV

ICD-10: Schädlicher Gebrauch

A. Nachweis, dass der Substanzgebrauch verantwortlich ist für die körperlichen oder psychischen Probleme, einschließlich der eingeschränkten Urteilsfähigkeit oder des gestörten Verhaltens, das eventuell zu Behinderung oder zu negativen Konsequenzen in den zwischenmenschlichen Beziehungen geführt hat.

B. Die Art der Schädigung sollte klar bezeichnet werden.

C. Das Gebrauchsmuster besteht mindestens seit 1 Monat oder trat wiederholt in den letzten zwölf Monaten auf.

D. Auf die Störung treffen die Kriterien einer anderen psychischen oder Verhaltensstörung bedingt durch dieselbe Substanz zum gleichen Zeitpunkt nicht zu (außer akute Intoxikation F1x.0).

DSM-IV: Substanzmissbrauch

A. Ein unangepasstes Muster von Substanzgebrauch führt in klinisch bedeutsamer Weise zu Beeinträchtigungen oder Leiden, wobei sich mindestens eines der folgenden Kriterien innerhalb desselben 12-Monats-Zeitraumes manifestiert.

1. Wiederholter Substanzgebrauch, der häufig zu einem Versagen bei der Arbeit, in der Schule oder zu Hause führt.

2. Wiederholter Substanzgebrauch in Situationen, in denen es aufgrund des Konsums zu einer körperlichen Gefährdung kommen kann.

3. Wiederkehrende Probleme mit dem Gesetz im Zusammenhang mit dem Substanzgebrauch.

4. Fortgesetzter Substanzgebrauch trotz ständiger oder wiederholter sozialer oder zwischenmenschlicher Probleme, die durch die Auswirkungen der psychotropen Substanz verursacht oder verstärkt werden.

B. Die Symptome haben niemals die Kriterien für Substanzabhängigkeit der jeweiligen Substanzklasse erfüllt. 
Tab. 2 Kriterien des Abhängigkeitssyndroms in der ICD-10

\section{ICD-10: Abhängigkeitssyndrom}

A. Ein starker Wunsch oder eine Art Zwang, psychotrope Substanzen zu konsumieren.

B. Verminderte Kontrollfähigkeit bezüglich des Beginns, der Beendigung und der Menge des Konsums.

C. Ein körperliches Entzugssyndrom bei Beendigung oder Reduktion des Konsums, nachgewiesen durch die substanzspezifischen Entzugssymptome oder durch die Aufnahme der gleichen oder einer nahe verwandten Substanz, um Entzugssymptome zu vermindern oder zu vermeiden.

D. Toleranzentwicklung gegenüber den Substanzeffekten. Es sind größere Mengen notwendig, um den gewünschten Effekt zu erreichen.

E. Einengung auf den Substanzgebrauch (Aufgabe oder Vernachlässigung anderer wichtiger Vergnügen oder Interessensbereiche wegen des Substanzgebrauchs).

F. Anhaltender Substanzgebrauch trotz eindeutig schädlicher Folgen.

schiedlichen interkulturellen Bedeutung nicht aufgenommen und stattdessen wurde auf deren Erfassung im Rahmen des multiaxialen diagnostischen Ansatzes verwiesen [15].

Bei der Definition des Abhängigkeitssyndroms kommt in beiden Systemen bei der psychischen Abhängigkeit als entscheidendes Kriterium das typische Beschaffungs- bzw. Einnahmeverhalten zur Anwendung, während die körperliche Abhängigkeit im Wesentlichen über das Entzugssyndrom definiert wird. In beiden Systemen kann die Diagnose eines Missbrauchs, einer Abhängigkeit oder einer anderen substanzinduzierten Störung auf jede dort definierte Substanzklasse angewendet werden.

Mit dieser operationalen Differenzierung von Missbrauch und Abhängigkeit ist auch die Bestimmung ihrer qualitativen Ausprägung über Screeninginstrumente möglich (z.B. Fagerström-Test für Nikotinabhängigkeit [16]; Lübecker Alkoholismus-Test [17]). In den Klassifikationssystemen selbst wird jedoch eine operationalisierte Schweregraddefinition nicht verankert.

Auch die weiteren unmittelbar suchtassoziierten Störungen werden in der ICD-10 und dem DSM-IV abgesehen von wenigen Ausnahmen weitgehend analog definiert (vgl. Tab. 3). So geben etwa ICD-10 (auf der Ebene der Forschungskriterien) und DSM-IV detaillierte substanzspezifische Symptome für Intoxikation und Entzug an, die sich weitestgehend entsprechen. Eine Ausnahme bildet in der ICD-10 lediglich die Verschlüsselung von Phencyclidin, das unter der „Restkategorie“ F19 klassifiziert wird.

Die in der ICD-10 abgebildeten substanzinduzierten psychotischen Störungen (F1x.5 und F1x.7) sind im DSM-IV anders verteilt. Die in der ICD-10 definierten psychotischen Störungen bei Substanzgebrauch (F1x.5) sind als psychotische Störungen mit Wahn oder Halluzinationen zu verstehen, nichtpsychotische affektive Störungen durch Substanzgebrauch sind als residualaffektive Zustandsbilder (F1x.72) zu klassifizieren.

\section{Zum Problem der Komorbidität}

In der ICD-10 wurde das Prinzip der Komorbidität verwirklicht, was zu einer substanziellen Zunahme nicht nur suchtbezogener Diagnosen, sondern auch weiterer psychischer Störungen geführt hat. Bereits Anfang der 90er Jahre konnte in der „Epidemiological Catchment Area Study“ "6] und dem „National Comorbidity Survey“ [18] gezeigt werden, dass in klinischen und epidemiologischen Stichproben das Ausmaß der Komorbidität zwischen Suchterkrankungen und anderen psychischen Störungen als ausgesprochen hoch einzuschätzen ist. Für bestimmte diagnostische Gruppen ergeben sich dabei spezifische und z.T. differenzialindikative Interventionen bei der Behandlungsbedürftigkeit (z.B. $[9,19,20]$ ). Auch für die Bundesrepublik sind die frühen Entwicklungsstufen für die Entstehung und den Verlauf bei jugendlichen Konsumenten gut belegt [21].

Am besten belegt sind komorbide Zusammenhänge mit Angststörungen, affektiven Störungen, psychotischen Erkrankungen und Persönlichkeitsstörungen, wobei aus methodischen Gründen erhebliche Streubreiten auftreten [8]. So ergab sich z.B. in der ICD-10-Merkmalslistenstudie neben hoher interner Komorbidität von Störungen durch psychotrope Substanzen weiterhin ein häufiges Auftreten dieser Störungen als Zweit- und Drittdiagnose [22,23].

Für differenzialindikative Prozesse sind die Wechselwirkungseffekte individuell zu spezifizieren, wobei u.a. Schuckit [24] am Beispiel der Komorbidität zwischen Alkoholabhängigkeit und affektiven Störungen fünf Möglichkeiten des Zusammenspiels aufgezeigt hat:

1. Alkohol kann depressive Symptome verursachen.

2. Vorübergehende depressive Symptome können längeren Trinkepisoden folgen.

3. Der Alkoholkonsum kann aufgrund primär affektiver Episoden ansteigen (z. B. bei Manie).

4. Depressive Symptome und Alkoholismus können bei anderen psychiatrischen Störungen zusammen auftreten.

5. Ein kleiner Teil der Patienten weist Alkoholabhängigkeit und affektive Störungen voneinander getrennt auf.

Weder in der ICD-10 noch im DSM-IV wird die Abbildung komorbider Störungen nach inhaltlichen Prinzipien verankert, d.h., es wird auf zeitbezogene (primäre vs. in ihrem zeitlichen Auftreten sekundäre Störung) oder ätiopathogenetische Ordnungsgesichtspunkte zugunsten einer rein deskriptiven Beschreibung verzichtet.

\section{Multiaxiale Systeme}

Empirische Studien haben sich bisher nur sehr eingeschränkt der vorliegenden multiaxialen Systeme bedient und in erster Linie Achse-I- und Achse-II-Störungen untersucht. Während die Global Assessment Functioning Scale des DSM-III und DSM-III-R noch vergleichsweise häufig eingesetzt wurde, wurde das multiaxiale System der ICD-10 (vgl. Tab.4) bzw. noch komplexere Konstrukte kaum untersucht, obgleich ihre Bedeutung für Fragen der Dokumentation und Qualitätssicherung erheblich sein dürften. So werden in dem multiaxialen System der ICD-10 wie im DSM-IV allerdings mit unterschiedlichen Skalenbildungen neben der Komorbidität das psychosoziale Funktionsniveau sowie störungsauslösende 
Tab. 3 Gegenüberstellung diagnostischer Kategorien in ICD-10 und DSM-IV [33]

\begin{tabular}{ll} 
ICD-10 DSM-IV & DS \\
\hline
\end{tabular}

F10.xx Störungen durch Akohol

F11.xx Störungen durch Opioide

F12.xx Störungen durch Cannabinoide

F13.xx Störungen durch Sedativa/Hypnotika

F14.xx Störungen durch Kokain

F15.xx Störungen durch sonstige Stimulantien einschließlich Koffein

F16.xx Störungen durch Halluzinogene

F17.xx Störungen durch Tabak

F18.xx Störungen durch flüchtige Lösungsmittel

F19.xx Störungen durch multiplen Substanzgebrauch und Konsum sonstiger psychotroper Substanzen

Mit der 4. und 5. Stelle können die klinischen Zustandsbilder näher bezeichnet werden:

.0 akute Intoxikation

.00 ohne Komplikation

.01 mit Verletzungen oder anderer körperlicher Schädigung

.02 mit anderen medizinischen Komplikationen

.03 mit Delir

.04 mit Wahrnehmungsstörungen

\section{.05 mit Koma}

.06 mit Krampfanfällen

.07 pathologischer Rausch

.1 schädlicher Gebrauch

\section{.2 Abhängigkeitssyndrom}

.20 gegenwärtig abstinent

.21 gegenwärtig abstinent, aber in beschützender Umgebung

.22 gegenwärtige Teilnahme an einem ärztlich überwachten Ersatzdrogenprogramm (kontrollierte Abhängigkeit)

.23 gegenwärtig abstinent, aber in Behandlung mit aversiven oder hemmenden Medikamenten

.24 gegenwärtiger Substanzgebrauch (aktive Abhängigkeit)

.25 ständiger Substanzgebrauch

.26 episodischer Substanzgebrauch (z. B. Dipsomanie)
... Alkohol

... Opiate

... Cannabis

... Sedativa, Hypnotika oder anxiolytikaähnliche Substanzen

... Kokain

... Amphetamine (amphetaminähnliche Substanzen und Koffein)

... Halluzinoge

... Nikotin

... Inhalanzien

... Phencyclidin (phencyclidinähnliche Substanzen), multiple

Substanzen, andere (unbekannte) Substanzen

303.00 Alkoholintoxikation

305.90 Koffeinintoxikation

292.89 Amphetamin-, Cannabis-, Kokain-, Halluzinogen-, Inhalanzien-, Opiat-, Phencyclidin-, Sedativa-, Hypnotika-, oder Anxiolytika-, andere (oder unbekannte) Substanzintoxikation

keine Entsprechung

keine Entsprechung

291.00 Alkoholintoxikationsdelir

282.81 Amphetamin-, Cannabis-, Kokain-, Halluzinogen-, Inhalanzien-, Opiat-, Phencyclidin-, Sedativa-, Hypnotika-, oder Anxiolytika-, anderes (oder unbekanntes) Substanzintoxikationsdelir

292.89 Amphetamin-, Cannabis-, Kokain-, Opiat-, Phencyclidin-, andere (oder unbekannte) Substanzintoxikation mit Wahrnehmungsstörungen

keine Entsprechung

keine Entsprechung

keine Entsprechung

305.00 Alkoholmissbrauch

305.70 Amphetaminmissbrauch

305.20 Cannabismissbrauch

305.60 Kokainmissbrauch

305.30 Halluzinogenmissbrauch

305.50 Opiatmissbrauch

305.40 Sedativa-, Hypnotika- oder Anxiolytikamissbrauch

305.90 Missbrauch durch Inhalanzien, Phencyclidin und andere (oder) unbekannte Substanzen

Abhängigkeit von:

303.90 Alkohol

305.10 Nikotin

304.40 Amphetaminen

304.30 Cannabis

304.20 Kokain

304.60 Inhalanzien

304.20 Opiaten

304.50 Halluzinogenen

304.90 Phencyclidin

304.10 Sedativa, Hypnotika oder Anxiolytika

304.80 Polytoxikomanie

304.90 von anderen (oder unbekannten) Substanzen Spezifikation:

- mit körperlicher Abhängigkeit

- ohne körperliche Abhängigkeit

- früh vollremittiert

- früh teilremittiert

- anhaltend vollremittiert

- anhaltend teilremittiert

- bei agonistischer Therapie

- in geschützter Umgebung 
Tab. 3 Gegenüberstellung diagnostischer Kategorien in ICD-10 und DSM-IV (Fortsetzung)

ICD-10

DSM-IV

\section{.3 Entzugssyndrom}

.30 unkompliziert

31 mit Krampfanfällen

.4 Entzugsyndrom mit Delir

.40 ohne Krampfanfälle

.41 mit Krampfanfällen

.5 psychotische Störung

.50 schizophreniform

.51 vorwiegend wahnhaft

.52 vorwiegend halluzinatorisch (einschließlich Alkoholhalluzinose)

.53 vorwiegend polymorph

.54 vorwiegend depressive Symptome

.55 vorwiegend manische Symptome

.56 gemischt

.6 amnestisches Syndrom

.7 Restzustand und verzögert auftretende psychotische Störung 70 Nachhallzustände (Flashbacks)

71 Persönlichkeits- oder Verhaltensstörung

.72 residualaffektives Zustandsbild

\section{.73 Demenz}

.74 andere anhaltende kognitive Beeinträchtigungen

.75 verzögert auftretende psychotische Störung

.8 sonstige psychische oder Verhaltensstörungen

.9 nicht näher bezeichnete psychische oder Verhaltensstörung
291.8 Alkoholentzug

292.0 Kokain-, Amphetamin-, Nikotin- Opiat-, Sedativa-, Hypnotikaoder Anxiolytika-, anderer (oder unbekannter) Substanzentzug

keine Entsprechung

keine Entsprechung

291.0 Alkoholintoxikationsdelir

292.81 Sedativa-, Hypnotika-, oder Anxiolytikaintoxikationsdelir

keine Entsprechung

keine Entsprechung

291.x Alkoholinduzierte psychotische Störung

.5 mit Wahn

3 mit Halluzinationen

292.xx Psychotische Störung induziert durch Amphetamine, Cannabis Kokain, Halluzinogene, Inhalanzien, Opiate, Phencyclidin, Sedativa, Hypnotika, Anxiolytika, andere (unbekannte) Substanze.11 mit Wahn.12 mit Halluzinationen

291.8 Alkoholinduzierte affektive Störung

292.84 Affektive Störung durch Amphetamine, Cannabis, Kokain, Halluzinogene, Inhalanzien, Opiate, Phencyclidin, sedativa, Hypnotika, Anxiolytika, andere (unbekannte) Substanzen - mit depressiven Merkmalen - mit manischen Merkmalen - mit gemischten Merkmalen

291.1 Persistierende alkoholinduzierte amnestische Störung

292.82 Persistierende amnestische Störung durch Sedativa, Hypnotika, Anxiolytika, andere (unbekannte) Substanzen

292.89 Persistierende Wahrnehmungsstörung im Zusammenhang mit Halluzinogenen (Flashbacks)

keine Entsprechung

292.84 Affektive Störung durch Amphetamine, Cannabis, Kokain, Halluzinogene, Inhalanzien, Opiate, Phencyclidin, sedativa, Hypnotika, Anxiolytika, andere (unbekannte) Substanzen - mit depressiven Merkmalen

- mit manischen Merkmalen

- mit gemischten Merkmalen

291.2 Persistierende alkoholinduzierte Demenz

292.83 Persistierende amnestische Störung durch Sedativa, Hypnotika, Anxiolytika, andere (unbekannte) Substanzen

keine Entsprechung

keine Entsprechung

291.8 Alkoholinduzierte Angststörung, Schlafstörung, sexuelle Funktions-Störung

292.89 Angststörung induziert durch Amphetamine, Koffeine, Cannabis, Kokain, Halluzinogen, Inhalanzien, Phencyclidin, Sedativa, Hypnotika, Anxiolytika, andere (oder) unbekannte Substanzen

292.89 Sexuelle Funktionsstörungen induziert durch Amphetamine, Kokain, Opiate, Sedativa, Hypnotika, Anxiolytika, andere (oder) unbekannte Substanzen

292.89 Schlafstörungen induziert durch Amphetamin, Koffein, Kokain, Opiate, Sedativa, Hypnotika, Anxiolytika, andere (oder) unbekannte Substanzen

291.9 Nicht näher bezeichnete Störung im Zusammenhang mit Alkohol

292.9 Nicht näher bezeichnete Störungen induziert durch Amphetamine, Koffein, Cannabis, Kokain, Halluzinogene, Inhalanzien, Phencyclidin, Sedativa, Hypnotika, Anxiolytika, andere (oder) unbekannte Substanzen 
und -aufrechterhaltende Faktoren auf separaten Achsen verankert. Im Hinblick auf die ICD-10 ergibt sich z.B. die Möglichkeit, auf der Achse III „Psychosoziale Belastungsfaktoren" entsprechende Kodierungen im Zusammenhang mit psychotropen Substanzen vorzunehmen (z.B. Z72 Probleme bei der Lebensführung: Z72.0 „Rauchen“, Z72.1 „Alkoholmissbrauch“). Darüber hinaus bestehen weitere Möglichkeiten, Z-Kodierungen in diesem Kontext zu nutzen (vgl. [11]): Z49.1 „Rehabilitation nach Alkoholabhängigkeit“ oder Z81 „Familienanamnese mit Hinweisen auf psychische und Verhaltensstörungen (Z81.2 „Familienanamnese mit Hinweisen auf Alkoholmissbrauch“).

Vereinzelt liegen Arbeiten zur psychischen Abwehr Alkoholabhängiger vor (John, 1990). Mit dem seit 1996 vorliegenden multiaxialen System zur Operationalisierten Psychodynamischen Diagnostik (vgl. Tab.5) wurden demgegenüber zwischenzeitlich einige Untersuchungen publiziert [26-28], eine entsprechende multizentrische Arbeitsgruppe hat sich mittlerweile etabliert. Das multiaxiale System der OPD erfasst dabei auf unterschiedlichen Achsen Krankheitserleben und Behandlungsvoraussetzungen, Beziehungs-, Konflikt- und Strukturdiagnostik sowie als Achse V ICD-10-Diagnostik [29-31].

Damit gilt auch für den Suchtbereich, dass eine multiaxiale, d.h. verschiedene Betrachtungsebenen integrierende klassifikatorische Diagnostik kaum ausreichend integriert wurde [32].

\section{Ausblick}

Mit der Veröffentlichung der operationalisierten Klassifikationssysteme ICD-10 und DSM-IV hat die kategoriale Klassifikation von Suchterkrankungen zweifelsohne einen bedeutsamen Fortschritt gemacht. Die klinisch relevanteste interne Schwäche dieser Systeme dürfte allerdings darin bestehen, dass sie keine explizit operationalisierten Schweregradbestimmungen erlauben und Komorbiditätsmuster rein deskriptiv ohne spezifizierte hierarchische oder ätiopathogenetische Reihung erfassen. Damit sind sie allein betrachtet für die Therapieplanung und die Indikation für bestimmte Interventionsformen nur unzureichend brauchbar. Für die Schweregradunterteilung werden in Zukunft, wie in anderen diagnostischen Feldern auch, kategoriale und dimensionale Verfahren kombiniert werden müssen, um zu reliablen und validen Ansätzen zu gelangen [32]. Eine angemessenere Abbildung der Komorbidität setzt darüber hinaus wahrscheinlich die Reintegration ätiologischer Vorannahmen in die deskriptive Diagnostik voraus, wie sie etwa mit der Operationalisierten Psychodynamischen Diagnostik verwirklicht wurden. Von einer indikativen Kopplung bestimmter Diagnosen mit spezifischen therapeutischen Interventionen sind wir in diesem Bereich, wie auch bei den meisten anderen psychischen Störungen, weit entfernt. Diese werden sich nur in Kombination mit dimensionalen und multiaxialen diagnostischen Ansätzen, die andere Merkmalsbereiche erfassen, entwickeln lassen. Dies war aber auch nicht der Anspruch, unter dem die operationalisierte Diagnostik nach ICD-10 und DSM-IV angetreten ist, denn erreicht werden sollten in einem ersten Schritt die methodischen Voraussetzungen, unter denen überhaupt sinnvolle klinische und wissenschaftliche Arbeit möglich wird: Kommunizierbarkeit und Reliabilität.

Tab. 4 Das multiaxiale System der ICD-10 [34]

Achse I Psychische Störungen und körperliche Erkrankungen

la psychische Störungen

lb Persönlichkeitsstörungen

Ic Störungen durch psychotrope Substanzen

Id körperliche Störungen

Achse II Beurteilung der sozialen Funktionseinschränkung (WHO Disablement Scale)

Ila Selbstfürsorge (Körperhygiene, Kleidung, Ernährung usw.)

Ilb Beruf (bezahlte Arbeit, Studium, Hausarbeit usw.)

Ilc Familie und Haushalt (Interaktion mit dem (Ehe-)Partner, Eltern, Kindern, und anderen Verwandten)

IId Funktionsfähigkeit im Weiteren sozialen Kontext (Beziehung zu Gemeindemitgliedern, Teilnahme an Freizeit- und sozialen Aktivitäten)

Ile Globaleinschätzung (Gesamtbeeinträchtigung)

Achse III Psychosoziale Belastungsfaktoren

Ereignisse und Merkmale aus folgenden Bereichen:

1. negative Erlebnisse in der Kindheit

2. Erziehung und Bildung

3. primäre Bezugsgruppe einschließlich Familie

4. soziale Umgebung

5. Wohnungsbedingungen und finanzielle Verhältnisse

6. Berufstätigkeit und Arbeitslosigkeit

7. Umweltbelastungen

8. psychosoziale oder juristische Probleme

9. Krankheiten oder Behinderungen in der Familie

10. Lebensführung/Lebensbewältigung 
Tab. 5 Das multiaxiale diagnostische System zur Operationalisierten Psychodynamischen Diagnostik (OPD)

Achse I Krankheitserleben und Behandlungsvoraussetzungen (4-stufige Fremdeinschätzung) von 1 (= niedriger) bis $\mathbf{4}$ (= hoher Ausprägungsgrad)

1. Beurteilung des Schweregrades der somatischen Erkrankung

2. Beurteilung des Schweregrades der psychischen Erkrankung

3. Leidensdruck

4. Beeinträchtigung des Selbsterlebens

5. Ausmaß der körperlichen Behinderung

6. sekundärer Krankheitsgewinn

7. Einsichtsfähigkeit in psychodynamische Zusammenhänge

8. Einsichtsfähigkeit für somatopsychische Zusammenhänge

9. Einschätzung der geeigneten Behandlungsform (Psychotherapie)

10. Einschätzung der geeigneten Behandlungsform (körperliche Behandlung)

11. Motivation zur Psychotherapie

12. Motivation zur körperlichen Behandlung

13. Compliance

14. Symptomdarbietung: somatische Symptomatik steht im Vordergrund

15. Symptomdarbietung: psychische Symptomatik steht im Vordergrund

16. psychosoziale Integration

17. persönliche Ressourcen

18. soziale Unterstützung

19. Angemessenheit der subjektiven Beeinträchtigung zum Ausmaß der Erkrankung

Achse II Beziehung (dysfunktionelles habituelles Beziehungsverhalten; Fremdeinschätzung von jeweils 2 im Sinne interpersoneller Kreismodelle definierten, nach Relevanz gewichteten Merkmalen je Perspektive und Dimension)

1. Perspektive A: Das Erleben des Patienten mit den Dimensionen „Der Patient erlebt sich immer wieder so, dass er ...“” und „Der Patient erlebt andere immer wieder so, dass er ..."

2. Perspektive B: Das Erleben des Interviewers mit den Dimensionen „Der Untersucher erlebt den Patienten immer wieder so, dass er ...." und „Der Untersucher erlebt sich gegenüber dem Patienten immer wieder so, dass er ....“

3. Psychodynamische Formulierung des dysfunktionalen Beziehungsverhaltens (Option)

Achse III Konflikt (Fremdeinschätzung mit 4-stufigem Rating von $\mathbf{0}$ (= nicht vorhanden) bis 3 (= hoch) für jeden definierten Konflikt)

1. Abhängigkeit vs. Autonomie

2. Kontrolle vs. Unterwerfung

3. Versorgung vs. Autarkie

4. Selbstwertkonflikte (narzisstische Konflikte, Selbst- vs. Objektwert)

5. Über-Ich- und Schuldkonflikte (egoistische vs. prosoziale Tendenzen)

6. Ödipale und sexuelle Konflikte

7. Identitätskonflikte (Identität vs. Dissonanz)

8. fehlende Konflikt- und Gefühlswahrnehmung

9. Aktualkonflikte

Achse IV Struktur (Fremdeinschätzung mit 4-stufigem Rating von 1 (= gut integriert) bis 4 (desintegriert))

1. Selbstwahrnehmung

2. Selbststeuerung

3. Abwehr

4. Objektwahrnehmung

5. Kommunikation

6. Bindung

7. Gesamtniveau

Achse V Psychische und psychosomatische Störungen nach dem Kapitel V (F) der ICD-10

Achse Va Psychische Störungen

Achse Vb Persönlichkeitsstörungen (Kategorien F60 und F61 der ICD-10)

Achse Vc Somatische Erkrankungen (andere Kapitel der ICD-10)

\section{Literatur}

${ }^{1}$ Freyberger HJ, Schulte-Markwort E, Siebel U. Das Konzept der Störungen durch psychotrope Substanzen (F1). In: Dilling H, Schulte-Markwort E, Freyberger HJ (Hrsg). Von der ICD-9 zur ICD-10. Neue Ansätze in der Diagnostik psychischer Störungen in der Psychiatrie, Psychosomatik und Kinder- und Jugendpsychiatrie. Bern: Hans Huber, 1994: 95-102

${ }^{2}$ Krausz M. Überlegungen zum Diskurs über Diagnostik bei Patienten mit schädlichem Konsum psychotroper Substanzen. In: Bundesverband für stationäre Suchtkrankenhilfe, Martin
Beutel (Hrsg). Diagnose Sucht. Schriftenreihe Band IV. Geesthacht: Buss-Neuland-Verlagsgesellschaft, 2000

${ }^{3}$ Dilling H, Dittmann V, Freyberger HJ. ICD-10 field trial in German-speaking countries. Pharmacopsychiatry 1990; 23 (suppl.): 135-216

${ }^{4}$ Dittmann V, Dilling H, Freyberger HJ. Psychiatrische Diagnostik nach ICD-10 - klinische Erfahrungen bei der Anwendung. Bern: Hans Huber, 1992

${ }^{5}$ Freyberger HJ, Dilling H, Stieglitz RD. ICD-10 field trial of the Diagnostic Criteria for Research in German-speaking countries. Psychopathlogy 1996; 29: 257-314 
${ }^{6}$ Regier DA, Farmer ME, Rae DS, Locke BZ, Kesth SJ, Judd LL, Goodwin FK. Comorbidity of mental disorders with alcohol and other drug abuse. Results from the Epidemiological Catchment Area (ECA) study. Journal of the American Medical Association 1990; 264: 2511-2518

${ }^{7}$ Hapke U, Rumpf HJ, John U. Alkoholabhängigkeit und -mißbrauch im Allgemeinkrankenhaus. Förderung der Inanspruchnahme suchtspezifischer Versorgungsangebote. In: John U \& Deutsche Hauptstelle gegen die Suchtgefahren (Hrsg). Regionale Suchtkrankenversorgung. Konzepte und Kooperationen. Freiburg: Lambertus, 1997: 101-108

${ }^{8}$ Maier W, Linz M, Freyberger HJ. Komorbidität von Substanzabhängigkeitsstörungen und anderen psychischen Störungen. In: Soyka M, Möller HJ (Hrsg). Alkoholismus als psychische Störung. Berlin, Heidelberg: Springer, 1997: 75-93

${ }^{9}$ Krausz M, Müller-Thomsen T. Therapie von psychischen Störungen und Sucht-Konzepte für Diagnostik, Behandlung und Rehabilitation. Freiburg: Lambertus, 1994

${ }^{10}$ McLellan AT, Kushner H, Peters R, Smith I, Grissom G, Pettinata $\mathrm{H}$, Argeriou M. The fifth edition of the Addiction Severity Index: Historical critique and normative data. Journal of Substance Abuse Treatment 1992; 9: 199-213

${ }^{11}$ Dilling $\mathrm{H}$, Mombour $\mathrm{W}$, Schmidt $\mathrm{MH}$. Internationale Klassifikation psychischer Störungen. ICD-10, Kapitel V (F), Klinischdiagnostische Leitlinien. Bern: Huber, 1993; 2. Auflage

${ }^{12}$ Dilling H, Freyberger HJ. Taschenführer zur Klassifikation psychischer Störungen. Mit Glossar und diagnostischen Kriterien. ICD-10: DCR 10. Bern: Hans Huber, 2000

${ }^{13} \mathrm{Sa}$ H, Wittchen HU, Zaudig M. DSM-IV, Diagnostisches und Statistisches Manual Psychischer Störungen. Göttingen: Hogrefe, 1996

${ }^{14}$ Edwards G, Gross M. Alcohol dependence: provisional description of a clinical syndrome. British Medical Journal 1976; 1 : 2058-2061

${ }^{15}$ Michels R, Siebel U, Freyberger HJ, Stieglitz RD, Schaub RT, Dilling H. The multiaxial system of ICD-10: evaluation of a preliminary draft in a multicentric field trial. Psychopathology 1996; 29: 347-356

${ }^{16}$ Schoberger R, Kunze U, Schmeiser-Rieder A. Diagnostik und Therapie der Nikotinabhängigkeit. Versicherungsmedizin 1997; 49: $25-29$

${ }^{17}$ Rumpf HJ, Hapke U, Hill A, John U. Development of a screening questionnaire for the general hospital and general practices. Alcoholism: Clinical and experiemental research 1997; 21: 894-898

${ }^{18}$ Kessler RC, McGonagle KA, Zhao S, Nelson CB, Hughes M, Eshleman S, Wittchen HU, Kendler KS. Lifetime and 12-month prevalence of DSM-III-R psychiatric disorders in the United States: Results from the National Comorbidity Survey. Archives of General Psychiatry 1994; 51: 8-19

${ }^{19}$ Baving L, Olbrich H. Alcoholism and depression. European Addiction Research 1996; 2: 29-35

${ }^{20}$ Freyberger HJ, Drescher S, Dierse B, Spitzer C. Psychotherapeutic outcome of inpatients with neurotic and personality disorders with and without a benzodiazepine dependence syndrome. European Addiction Research 1996; 2: 53-61

${ }^{21}$ Wittchen HU, Nelson CB. Early developmental stages of substance abuse. European Addiction Research 1998; 4: 5-82

${ }^{22}$ Trabert W, Dittmann V, Mombour W. Störungen durch psychotrope Substanzen (Abschnitt F1). In: Dittmann V, Dilling H, Freyberger HJ (Hrsg). Psychiatrische Diagnostik nach ICD-10 klinische Erfahrungen bei der Anwendung. Bern: Hans Huber, 1992: 45-53

${ }^{23}$ Stieglitz RD, Schulte-Markwort E, Zaudig M. Die Komorbidität psychiatrischer Störungen in der ICD-10 Merkmalslistenstudie. In: Dittmann V, Dilling H, Freyberger HJ (Hrsg). Psychiatrische
Diagnostik nach ICD-10 - klinische Erfahrungen bei der Anwendung. Bern: Hans Huber, 1992: 111-119

${ }^{24}$ Schuckit MA. The relationship between alcohol problems, substance abuse and psychiatric syndromes. In: Pincus M (Hrsg). Soure book for DSM-IV. Washington DC: American Psychiatric Press, 1994; 1: 45-65

${ }^{25}$ John U. Psychische Abwehr Alkoholabhängiger. Erklärungsansätze, empirische Bestimmung und Behandlung. In: Schwoon D, Krausz M (Hrsg). Suchtkranke. Die ungeliebten Kinder der Psychiatrie. Stuttgart: Enke, 1990: 61-68

${ }^{26}$ Nitzgen D, Brünger M. Operationalisierte Psychodynamische Diagnostik in der Rehabilitationsklinik Birkenbuck: Einsatz und Befunde. In: Schneider W, Freyberger HJ (Hrsg). Was leistet die OPD? Empirische Befunde und klinische Erfahrungen mit der Operationalisierten Psychodynamischen Diagnostik. Bern: Hans Huber, 2000: 238-252

${ }^{27}$ Reymann G, Zbikowski A, Martin K, Tetzlaff M, Janssen PL. Erfahrungen mit der Anwendung von Operationalisierter Psychodynamischer Diagnostik bei Alkoholkranken. In: Schneider W, Freyberger HJ (Hrsg). Was leistet die OPD? Empirische Befunde und klinische Erfahrungen mit der Operationalisierten Psychodynamischen Diagnostik. Bern: Hans Huber, 2000: 229-237

28 Thomasius R, Kraus D, Sack PM, Wuchner B, Gemeinhardt B, Schindler A, Schuhbert C, Daporta-Albouy J, Heinz B. Zum Einsatz der Operationalisierten Psychodynamischen Diagnostik in der Suchtforschung: Erste Erfahrungen in Querschnitts- und Verlaufsuntersuchungen. In: Schneider W, Freyberger HJ (Hrsg). Was leistet die OPD. Empirische Befunde und klinische Erfahrungen mit der Operationalisierten Psychodynamischen Diagnostik. Bern: Hans Huber, 2000: 218-228

${ }^{29}$ Arbeitskreis OPD. Operationalisierte Psychodynamische Diagnostik. Grundlagen und Manual. Bern: Hans Huber, 1982; 2. korr. Auflage

${ }^{30}$ Schauenburg H, Freyberger HJ, Cierpka M, Buchheim P. OPD in der Praxis. Konzepte, Anwendungen, Ergebnisse der Operationalisierten Psychodynamischen Diagnostik. Bern: Hans Huber, 1998

${ }^{31}$ Schneider W, Freyberger HJ. Was leistet die OPD? Empirische Befunde und klinische Erfahrungen mit der Operationalisierten Psychodynamischen Diagnostik. Bern: Hans Huber, 2000

${ }^{32}$ Freyberger HJ, Stieglitz RD, Wittchen HU. Klassifikation. In: Stieglitz RD, Baumann U, Freyberger HJ (Hrsg). Psychodiagnostik in Klinischer Psychologie, Psychiatrie, Psychotherapie. Stuttgart: Thieme, 2001

${ }^{33}$ van Drimmelen-Krabbe J, Bertelsen A, Pull C. Gegenüberstellung von ICD-10 und DSM-IV. In: Helmchen H, Henn F, Lauter H, Sartorius N (Hrsg). Psychiatrie der Gegenwart. Band 2: Allgemeine Psychiatrie. 4. Auflage. Heidelberg, Berlin: Springer, 2000: 119-174

${ }^{34}$ Siebel U, Michels R, Freyberger HJ, Dilling H. Das multiaxiale System zum Kapitel V (F) der ICD-10. Unveröffentlichtes Manuskript, Klinik für Psychiatrie der Medizinischen Universität Lübeck 1997

\section{Prof. Dr. med. Harald J. Freyberger}

Klinik und Poliklinik für Psychiatrie und Psychotherapie der Ernst-Moritz-Arndt Universität Greifswald im Klinikum Stralsund Rostocker Chaussee 70 18437 Stralsund

E-mail: freyberg@rz.uni-greifswald.de 